\title{
Sports Economic Activities Income Forecasting based on Genetic Support Vector Regression Algorithm
}

\author{
G.U. Guoai*
}

Beijing WuZi University, Beijing, 101149, China

\begin{abstract}
In order to solve the shortcoming of support vector regression algorithm, sports economic activities income forecasting based on genetic support vector regression algorithm is presented in this paper. As the kernel parameter, insensitive loss parameter and penalty parameter have a great influence on the forecasting performance of support vector regression algorithm, genetic algorithm is used to perform the parameters optimization of support vector regression algorithm simultaneously in this study. The experimental results show that the sports economic activities income forecasting results of genetic support vector regression model trained by the four-dimension training samples are more excellent than those of genetic support vector regression model trained by the three-dimension training samples, and the sports economic activities income forecasting ability of genetic support vector regression model trained by the fourdimension training samples is better than that of support vector regression model trained by the four-dimension training samples. Thus, genetic support vector regression algorithm has a good application prospect in sports economic activities income forecasting.
\end{abstract}

Keywords: Economic activities income, Support vector regression algorithm, The sports.

\section{INTRODUCTION}

Support vector regression algorithm is a non-linear kernel function-based regression method. As the kernel parameter $\sigma$, insensitive loss parameter $\varepsilon$ and penalty parameter $C$ have a great influence on the forecasting performance of support vector regression algorithm, genetic algorithm is used to perform the parameters optimization of support vector regression algorithm simultaneously in this study $[1,2]$. In order to solve the shortcoming of support vector regression algorithm, sports economic activities income forecasting based on genetic support vector regression algorithm is presented in this paper. The dimension of the training samples has a great influence on the forecasting performance of genetic support vector regression algorithm. Thus, the three-dimension training samples and four-dimension training samples are used to train genetic support vector regression model respectively. It can be seen that the sports economic activities income forecasting results of genetic support vector regression model trained by the fourdimension training samples are more excellent than those of genetic support vector regression model trained by the threedimension training samples, and the sports economic activities income forecasting ability of genetic support vector regression model trained by the four-dimension training samples is better than that of support vector regression model trained by the four-dimension training samples.
Thus, genetic support vector regression algorithm has a good application prospect in sports economic activities income forecasting [3].

\section{GENETIC SUPPORT VECTOR REGRESSION ALGORITHM}

Support vector regression algorithm is a non-linear kernel function-based regression method, and the model of support vector regression algorithm can be described as follows:

$f(x)=w^{\prime} \phi(x)+b$

where $w$ is the weight and $b$ is the bias term.

The weight $w$ and the bias $b$ can be obtained by solving the following optimization problem:

$$
\min \left[\frac{1}{2}\|w\|^{2}+C \sum_{i=1}^{n} L\left(y_{i}, f(x)\right)\right]
$$

Subject to

$$
\left\{\begin{array}{l}
y_{i}-\left\langle w, \phi\left(x_{i}\right)\right\rangle-b \leq \varepsilon \\
\left\langle w, \phi\left(x_{i}\right)\right\rangle+b-y_{i} \leq \varepsilon
\end{array}\right.
$$

where $C$ is regularization parameter.

Then, the slack variables $\xi_{i}, \xi_{i}^{*}$ are introduced to generate the convex optimization problem: 
$\min \left[\frac{1}{2}\|w\|^{2}+C \sum_{i=1}^{n}\left(\xi_{i}+\xi_{i}^{*}\right)\right]$

Subject to

$\left\{\begin{array}{l}y_{i}-\left\langle w, \phi\left(x_{i}\right)\right\rangle-b \leq \varepsilon+\xi_{i}, \xi_{i} \geq 0 \\ \left\langle w, \theta\left(x_{i}\right)\right\rangle+b-y_{i} \leq \varepsilon+\xi_{i}^{*}, \xi_{i}^{*} \geq 0\end{array}\right.$

Finally, the model of support vector regression algorithm can be written as follows:

$$
f(x)=\sum_{i=1}^{n}\left(a_{i}-a_{i}^{*}\right) k\left(x_{i}, x\right)+b
$$

where $\alpha_{i}$ and $\alpha_{i}^{*}$ with non-zero values are the support vectors, and $k\left(x_{i}, x\right)$ is the kernel function $[4,5]$.

Radial basis function (RBF) is used to create the support vector regression model, which is defined as follows:

$$
k\left(x_{i}, x\right)=\exp \left(\left\|x_{i}-x\right\| / 2 \sigma^{2}\right)
$$

The training sample sets are established as follows:

$$
X=\left[\begin{array}{cccc}
b_{1} & b_{2} & \cdots & b_{l} \\
b_{2} & b_{3} & \cdots & b_{l+1} \\
\vdots & \vdots & & \vdots \\
b_{n-l} & b_{n-l+1} & \cdots & b_{n-1}
\end{array}\right] Y=\left[\begin{array}{c}
b_{1} \\
b_{2} \\
\vdots \\
b_{n}
\end{array}\right]
$$

where $l$ is the dimension of the input vector in the training sample sets.

As the kernel parameter $\sigma$, insensitive loss parameter $\varepsilon$ and penalty parameter $C$ have a great influence on the forecasting performance of support vector regression algorithm, genetic algorithm is used to perform the parameters optimization of support vector regression algorithm simultaneously in this study.

The operational process of genetic support vector regression algorithm can be shown in Fig. (1).

(1) Generate the initial population

The population is consisted of 20 chromosomes which is composed of the kernel parameter $\sigma$, insensitive loss parameter $\varepsilon$ and penalty parameter $C$. Randomly generate initial population of chromosomes.

(2) Evaluate the fitness of each chromosome

The training subsets and the fitness function are used to evaluate the fitness value of each chromosome.

\section{(3) Set up GA operators}

The new chromosomes are generated by GA operators, and the roulette wheel is used to select and reproduce, crossover with 0.5 rate is used to exchange the genes between two chromosomes, and mutation with 0.01 rate is used to alter binary code.

\section{(4) Judge the stopping criteria}

The evolutionary process proceeds until the stopping criteria is satisfied.

\section{EXPERIMENTAL RESULTS OF SPORTS ECONOMIC ACTIVITIES INCOME FORECASTING}

As shown in (Fig. 2), sports economic activities income data of China from 1991 to 2002 are applied to testify sports economic activities income forecasting ability of genetic support vector regression algorithm. The dimension of the training samples has a great influence on the forecasting

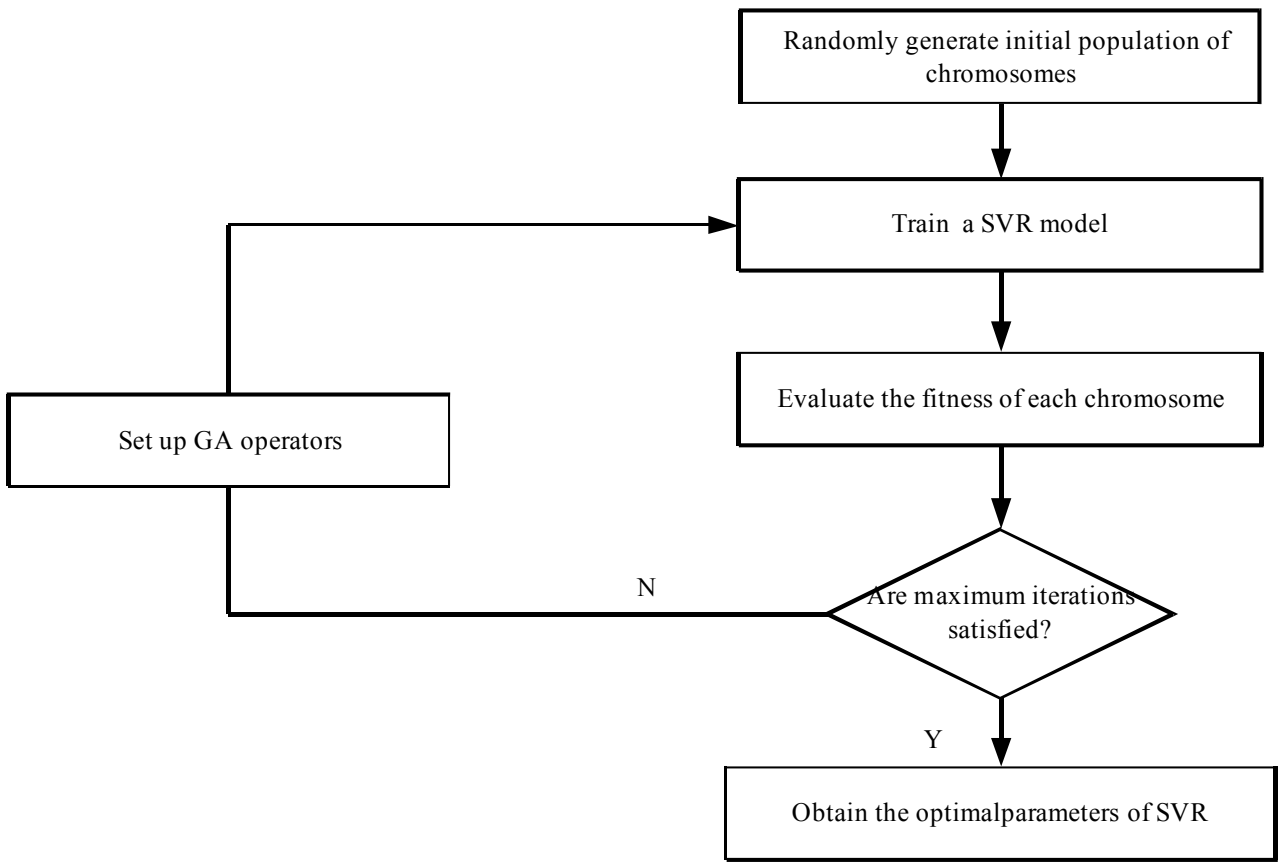

Fig. (1). The operational process of genetic support vector regression algorithm. 


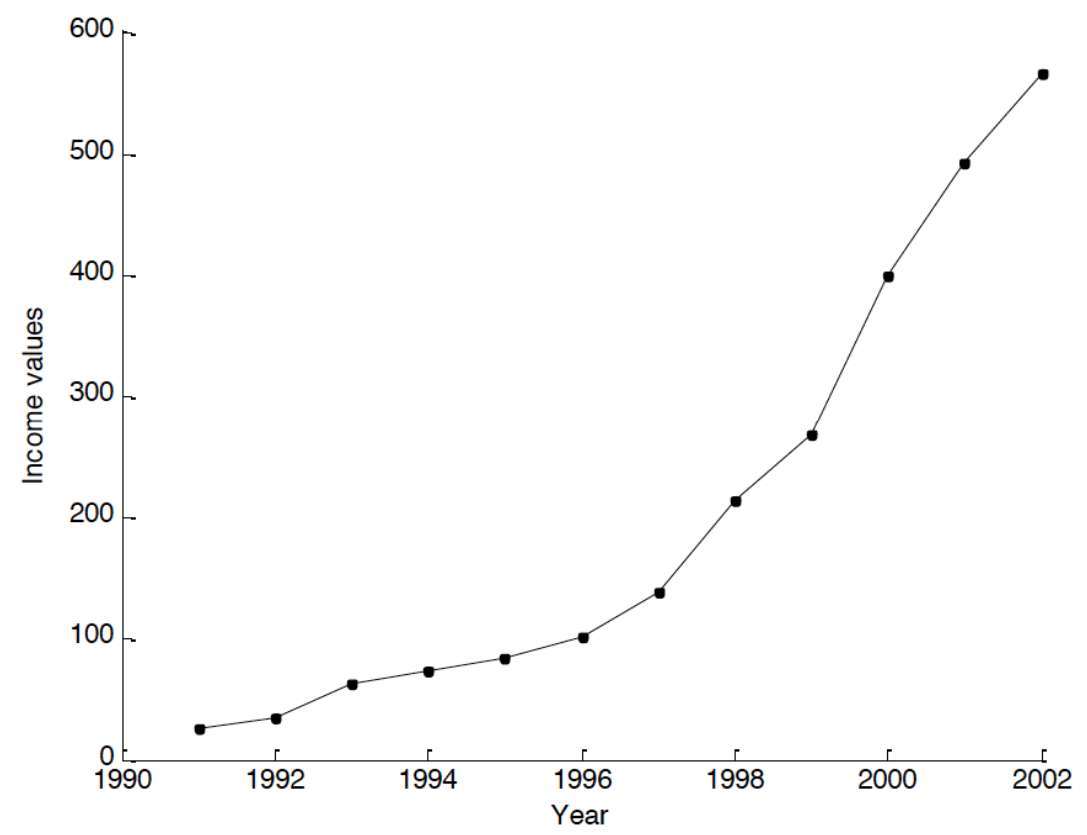

Fig. (2). Sports economic activities income data of China from 1991 to 2002.

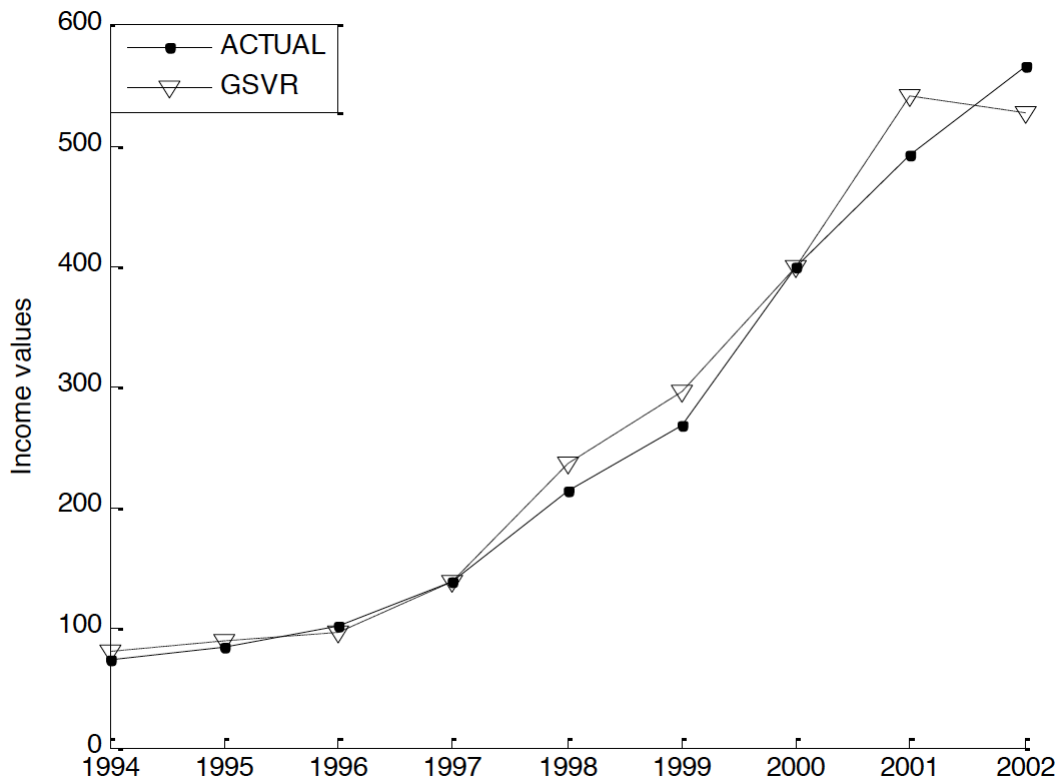

Fig. (3). The sports economic activities income forecasting results of genetic support vector regression model.

performance of genetic support vector regression algorithm. Thus, the three-dimension training samples are firstly used to train genetic support vector regression model [6-8].

The sports economic activities income forecasting results of genetic support vector regression model are shown in (Fig. 3), and the sports economic activities income forecasting results of support vector regression model are shown in (Fig. 4). It can be seen that the sports economic activities income forecasting ability of genetic support vector regression model trained by the three-dimension training samples is better than that of support vector regression model trained by the three-dimension training samples.
Then, the four-dimension training samples are firstly used to train support vector regression model. The sports economic activities income forecasting results of genetic support vector regression model are shown in (Fig. 5), and the sports economic activities income forecasting results of support vector regression model are shown in (Fig. 6). It can be seen that the sports economic activities income forecasting results of genetic support vector regression model trained by the four-dimension training samples are more excellent than those of genetic support vector regression model trained by the three-dimension training samples, and the sports economic activities income forecasting ability of genetic support vector regression 


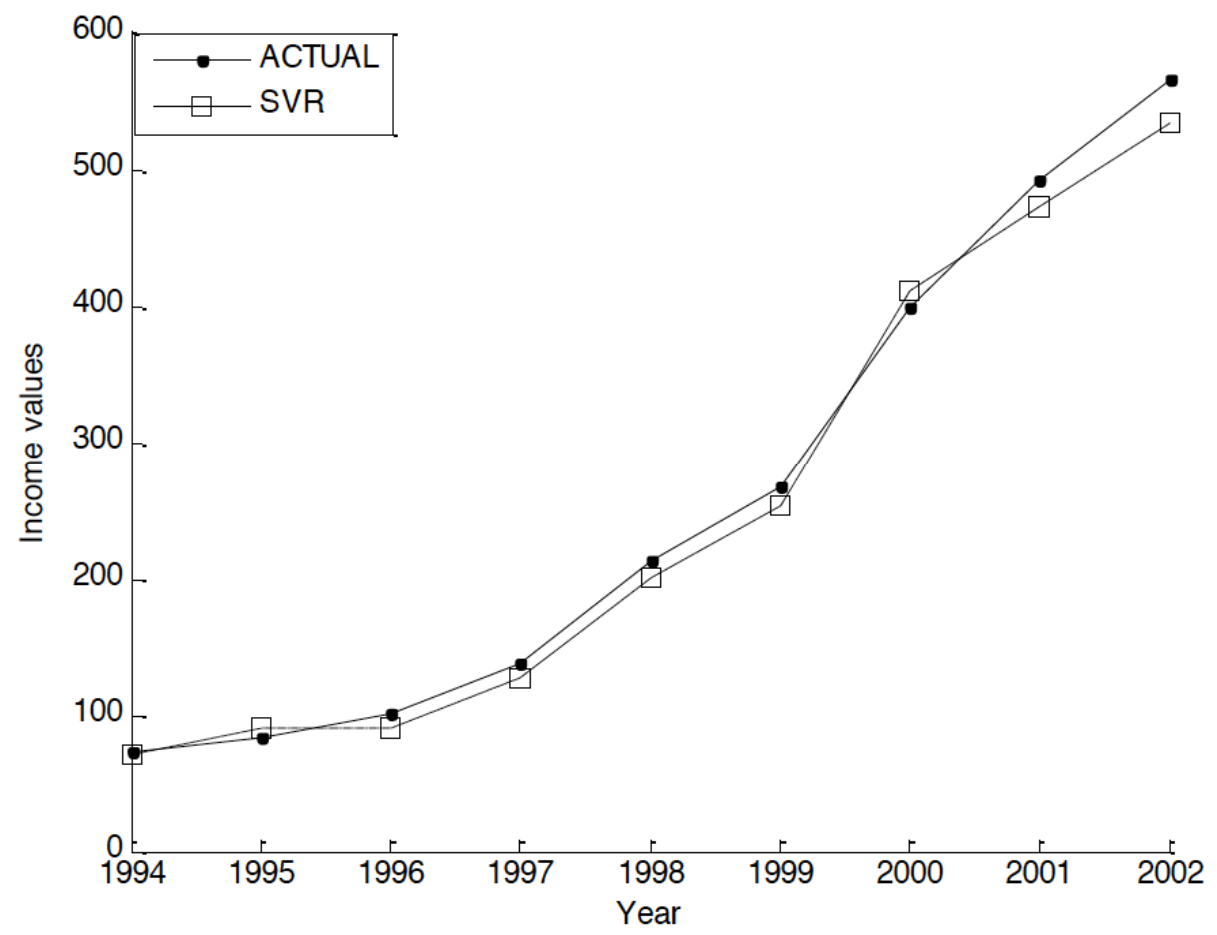

Fig. (4). The sports economic activities income forecasting results of support vector regression model.

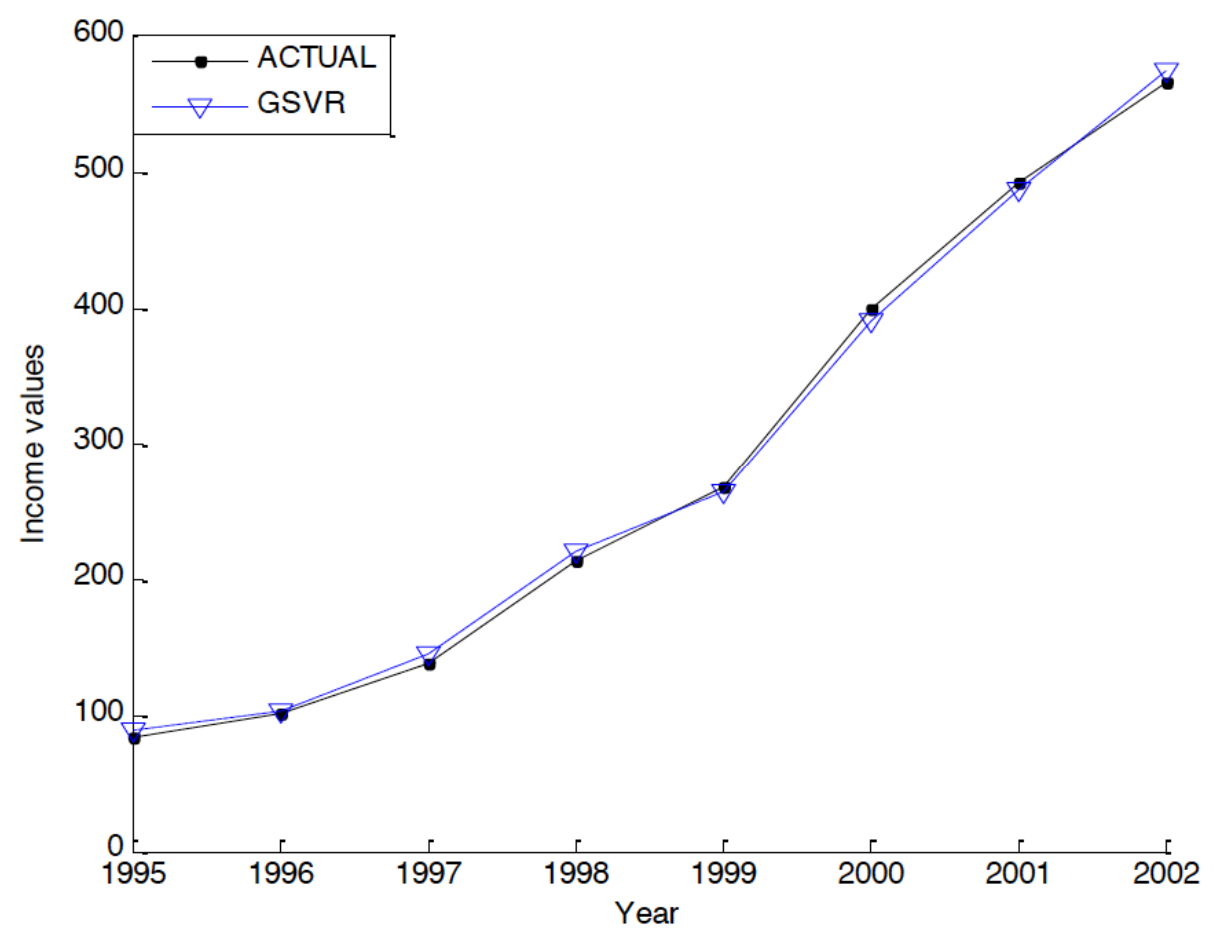

Fig. (5). The sports economic activities income forecasting results of genetic support vector regression model.

model trained by the four-dimension training samples is better than that of support vector regression model trained by the four-dimension training samples. Thus, genetic support vector regression algorithm has a good application prospect in sports economic activities income forecasting $[9,10]$.

\section{CONCLUSION}

In order to solve the shortcoming of support vector regression algorithm, sports economic activities income forecasting based on genetic support vector regression algorithm is presented in this paper. The experimental results 


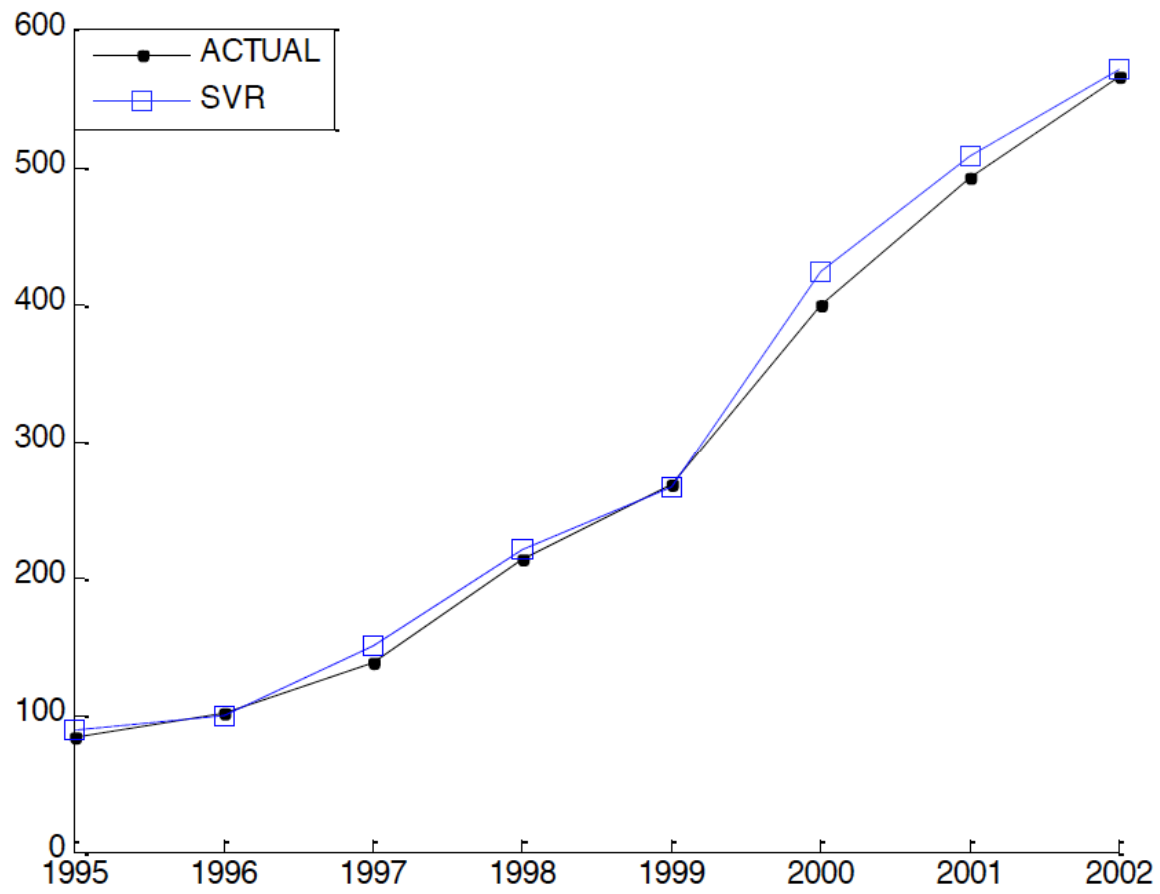

Fig. (6). The sports economic activities income forecasting results of support vector regression model.

show that the sports economic activities income forecasting results of genetic support vector regression model trained by the four-dimension training samples are more excellent than those of genetic support vector regression model trained by the three-dimension training samples, and the sports economic activities income forecasting ability of genetic support vector regression model trained by the fourdimension training samples is better than that of support vector regression model trained by the four-dimension training samples. Thus, genetic support vector regression algorithm has a good application prospect in sports economic activities income forecasting.

\section{CONFLICT OF INTEREST}

The author confirms that this article content has no conflict of interest.

\section{ACKNOWLEDGEMENTS}

Declared none.

\section{REFERENCES}

[1] B. R. Chang and H. F. Tsai, "Nested local adiabatic evolution for quantum-neuron-based adaptive support vector regression and its forecasting applications," Expert Systems with Applications, vol. 36, pp. 3388-3400, 2009.

[2] H. Yao and J. Chu, "Operational optimization of a simulated atmospheric distillation column using support vector regression models and information analysis," Chemical Engineering Research and Design, vol. 90, pp. 2247-2261, 2012.

[3] M. Han, and J. Yin, "The hidden neurons selection of the wavelet networks using support vector machines and ridge regression," Neurocomputing, vol. 72, pp. 471-479, 2008.

[4] P. Siarry, A. Pétrowski, and M. Bessaou, "A multipopulation genetic algorithm aimed at multimodal optimization," Advances in Engineering Software, vol. 33, pp. 207-213, 2002.

[5] H. Rahami, A. Kaveh, and Y. Gholipour, "Sizing, geometry and topology optimization of trusses via force method and genetic algorithm," Engineering Structures, vol. 30, pp. 2360-2369, 2008.

[6] N.-C. Chiu, S.-C. Fang, and Y.-S. Lee, "Sequencing parallel machining operations by genetic algorithms," Computers \& Industrial Engineering, vol. 36, pp. 259-280, 1999.

[7] A. Reese, "Random number generators in genetic algorithms for unconstrained and constrained optimization," Nonlinear Analysis: Theory, Methods \& Applications, vol. 71, pp. e679-e692, 2009.

[8] L. Zhu and W. Zhang, "A TOPSIS based approach to emergency material delivery using hybrid genetic algorithm," International Journal of Advancements in Computing Technology, vol. 4, no. 9, pp. 129-137, 2012.

[9] Y.-H. Chang, "Adopting co-evolution and constraint-satisfaction concept on genetic algorithms to solve supply chain network design problems," Expert Systems with Applications, vol. 37, pp. 69196930, 2010.

[10] H. R. Choi, B. K. Park, J. Lee, and C. Park, "Dispatching of container trucks using genetic algorithm," In: (ICIS), $4^{\text {th }}$ International Conference on Interaction Sciences, 2011, pp. 146-151. 